\title{
Prevotella copri associated with new-onset untreated RA
}

By parallel genetic sequencing the intestinal microbiome, researchers have discovered a high prevalence of the bacteria Prevotella copri in patients with rheumatoid arthritis (RA). "It has been suspected that RA was linked to infections or perhaps even diet, but this is the first finding of an association with a specific microbe," says Professor Dan Littman about his collaborative study now published in eLife. Previous work using mouse models of arthritis identified that intestinal microbiota can affect inflammation in the joints. Littman explains, "we sought to find out if something similar was going on in human disease."

By overpopulating the intestines of mice with segmented filamentous bacteria, researchers have previously induced intestinal type $17 \mathrm{~T}$ helper cells that Littman says "might circulate and contribute to systemic autoimmune diseases". For this reason his team wanted to characterize the intestinal microbiome of patients with new onset untreated RA (NORA). The researchers performed parallel sequencing of bacterial DNA from human faecal samples and detected $P$. copri in $75 \%$ (33 of 44 ) of patients with NORA and only in $21.4 \%$ ( 6 of 28 ) of healthy individuals (with a threshold for detection of $>5 \%$ abundance). Unexpectedly, they also found that only $11.5 \%$ ( 3 of 26 ) of patients with chronic RA ( $\geq 6$ months since diagnosis) tested positive for $P$. copri. Unlike patients with NORA, those with chronic RA were treated with DMARDs, biologic agents or steroids. Therefore, the researchers hypothesized in their report that there might be a NORA-specific systemic inflammatory response or, alternatively, that $P$. copri fail to thrive in the gut without inflammatory conditions.

Metagenomic analysis revealed that certain microbial genes were linked to the $P$. copri genomes in patients with NORA. Furthermore, the researchers found an inverse correlation between HLA susceptibility alleles in patients with NORA and the abundance of $P$. copri detected in their faecal samples. Littman hypothesizes that this is "consistent with P. copri in patients being pathogenic." Further supporting this view $P$. copri colonization exacerbated chemical colitis in mice; however, data showing a causal link between $P$. copri colonization of the gut and joint inflammation is still lacking. Littman concludes, "these remain correlations, and future studies need to be focused on mechanisms, to determine if $P$. copri has a pathogenic function.”

Nicholas J. Bernard

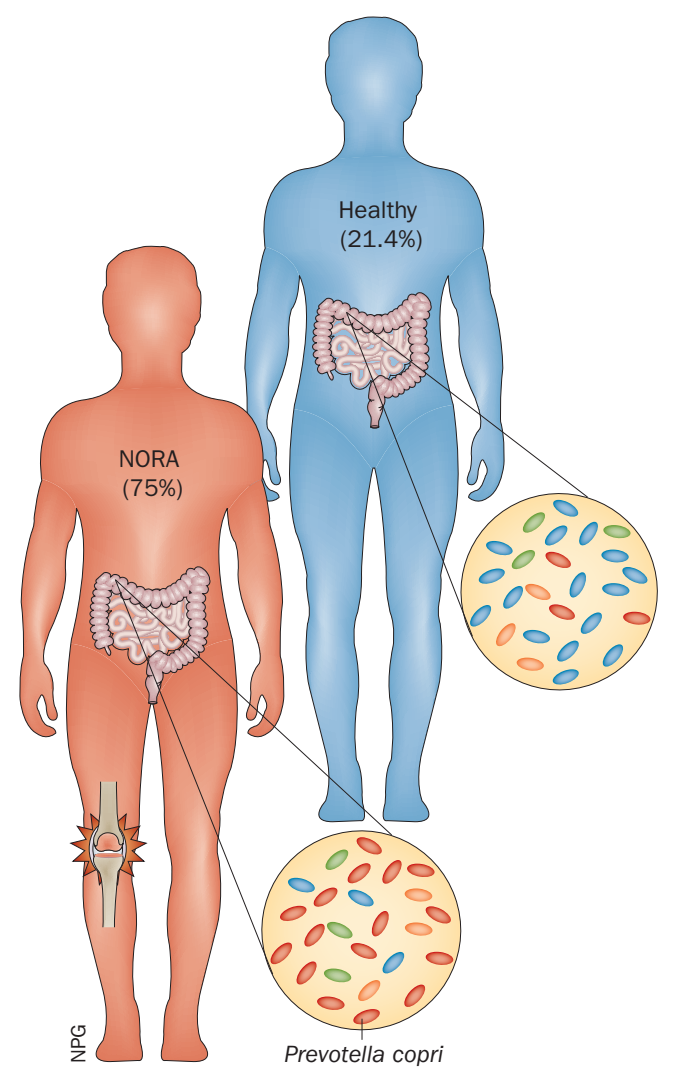

Original article Scher, J. U. et al. Expansion of intestinal Prevotella copri correlates with enhanced susceptibility to arthritis. eLife doi:10.7554/eLife.01202 